\title{
Multi-DOF Forceps Manipulator System for Laparoscopic Surgery
}

\author{
Ryoichi Nakamura $^{1}$, Etsuko Kobayashi ${ }^{2}$, Ken Masamune ${ }^{3}$, Ichiro Sakuma ${ }^{2}$, \\ Takeyoshi Dohi ${ }^{2}$, Naoki Yahagi ${ }^{2}$, Takayuki Tsuji ${ }^{2}$, Daijo Hashimoto ${ }^{4}$, \\ Mitsuo Shimada ${ }^{5}$, Makoto Hashizume \\ ${ }^{1}$ Dept. of Precision Engineering, Graduate School of Engineering, \\ ${ }^{2}$ Institute of Environmental Studies, Graduate school of Frontier Science, \\ the Univ. of Tokyo, 7-3-1, Hongo, Bunkyo-ku, Tokyo 113-8656 Japan \\ \{ryoichi, etsuko, sakuma, dohi, yahagi, tsuji\}@miki.pe.u- \\ tokyo.ac.jp \\ ${ }^{3}$ Dept. of Bio-Technology, College of Science and Engineering, Tokyo Denki University, \\ Ishizaka, Hatoyama-cho, Hiki, Saitama, 350-0395, Japan \\ masa@b.dendai.ac.jp \\ ${ }^{4}$ Department of Surgery, Saitama Medical Center, Saitama Medical School, \\ 1981, Tsujido, Kamoda, Kawagoe-shi, Saitama 350-8550 Japan \\ ${ }^{5}$ Department of Surgery and Science, Graduate School of Medical Sciences, \\ ${ }^{6}$ Dept. of Disaster and Emergency Medicine, Grad. School of Medical Science, \\ Kyushu University, 3-1-1, Maidashi, Higashi-ku, Hukuoka 812-8582 Japan \\ mshimada@surg2.med.kyushu-u.ac.jp \\ mhashi@dem.med.kyushu-u.ac.jp
}

\begin{abstract}
Many problems in laparoscopic surgery are due to the poor degrees of freedom of movement (DOF) in controlling forceps and laparoscopes. The Multi-DOF forceps manipulator we have newly developed has two additional DOF of bending on the tip of forceps, and provides new surgical fields and techniques for surgeons. The most remarkable characteristics of the prototype described in this paper are: 1) the small diameter $(\varphi 6 \mathrm{~mm})$ and the small radius of curvature of bending; 2) the large force generated on each bending and grasping axis; 3 ) the confirmation of perfect cleanness and sterilization of this manipulator. The effectiveness of these characteristics was confirmed in actual testing by surgeons. This manipulator can solve problems met with in laparoscopic surgery, and will establish new standards for laparoscopic surgery with higher effectiveness and safety.
\end{abstract}

\section{Introduction}

Laparoscopic surgery is now widely established as minimal invasive surgery (MIS). However, it has some problems and difficulties due to the "minimal invasive" method. The forceps and laparoscope are inserted into the visceral cavity through the trochars that are fixed on the abdominal wall. These tools have only four degrees of freedom of movement (DOF) and small working areas (Fig. 1).

Using conventional tools (forceps), surgeons can approach an operation point only through a single approach path. However, in some cases, various alternative approach 
paths for an operation area are needed. The tools thus require additional DOF to solve these problems (Fig. 2).
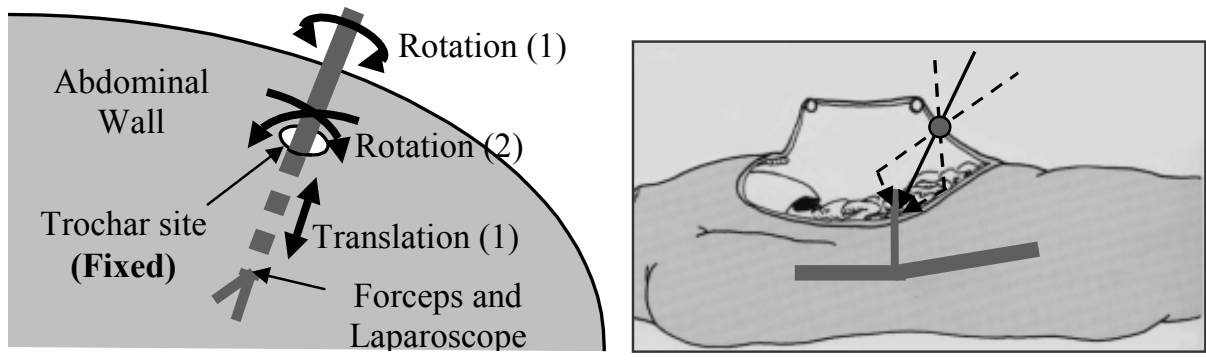

Fig. 1. Four DOF of surgical tools in laparoscopic surgery

Fig. 2. Approach path of surgical tools is approach path of conventional tools.
--- are approach paths of tools with additional DOF.

Surgical robots, which can help surgeons' manipulation such as steadier holding, more precise operating, and remote control, are being studied and developed. However, there are many problems in the use of robots in medical fields. The most important consideration must be safety, both in terms of engineering and medical effectiveness.

Recently, forceps with additional DOF for MIS have been studied in many institutes and applied to many actual cases [1-5], but in laparoscopic surgery, there are very few such cases. This is mainly due to the small force of additional DOF and reduced safety. We must develop new mechanisms with additional DOF, taking these considerations into account.

In this paper, we present a novel forceps manipulator with Multi-DOF. This manipulator has important features for laparoscopic surgery. The methods used in order to construct the forceps manipulator with the desired features are presented in Section 2. The experimental results are presented in Section 3, including the measurement of mechanical characteristics and the performance of this manipulator in surgical environments. Using this manipulator, problems in laparoscopic surgery are clearly solved, and new standards with higher effectiveness and safety are achieved.

\section{Method}

\subsection{Requirements}

When considering the need for increased force delivery and safety, and the basic requirement for new surgical tools for laparoscopic surgery, we defined the characteristics of the forceps we have developed as follows:

(1) DOF: two bending and one grasping (or scissoring or another DOF for end effector) on the tip of the forceps; 
(2) Radius of curvature of bending: as small as possible (for safety);

(3) Diameter of the forceps: about $5 \mathrm{~mm}$;

(4) Generated force: about $0.5 \mathrm{kgf}$ for bending / about $1 \mathrm{kgf}$ for grasping.

From the viewpoint of expanding the number of approach paths and the fields of operation of forceps, those with additional DOF and a large radius of curvature of bending would seem to be very useful. However, an instrument with a wide operating area also risks large and fatal errors if it is mishandled. Achieving large bends in the limited space of the visceral cavity is both difficult and dangerous. We decided to develop the first prototype with only two additional DOF and a small radius of curvature of bending.

It is difficult to determine the requirement of force generated accurately, because the measurement of the handling force of surgical tools in surgery is difficult. We decided the requirement from an experimental study using PVC [6].

\subsection{Bending Mechanism}

We developed the Multi-DOF forceps manipulator, which provides two additional DOF of bending. The bending mechanism is composed of four stainless steel rings with a coupling giving one DOF, stainless wire, and Teflon tubes (shown in Fig. 3 and 4). This mechanism is driven by four stainless steel wires (Fig. 5). The ranges of bending motion are 0 to 90 degrees for each two DOF.

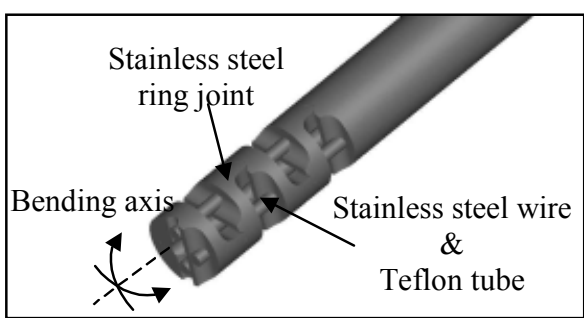

Fig. 4. Bending mechanism

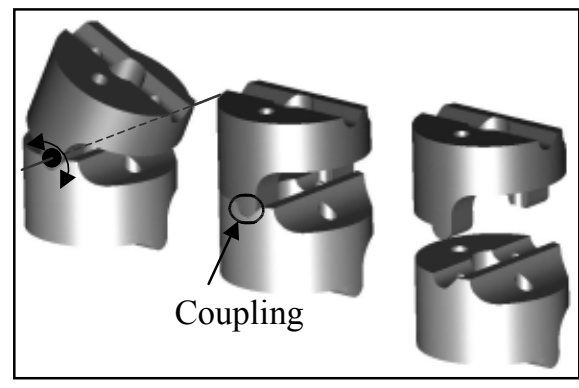

Fig. 3. Stainless steel ring joint

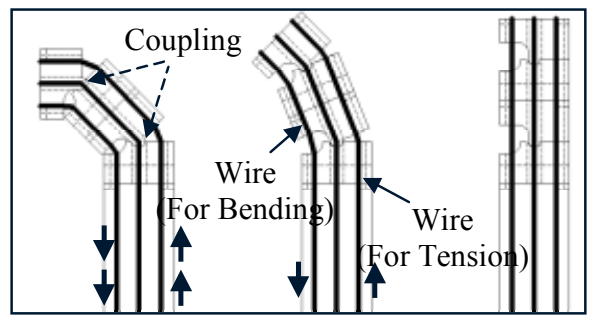

Fig. 5. Wire drive of bending mechanism

This mechanism is very simple, with high rigidity. It can easily be miniaturized. A wide range of bending provides a wide range of operations, but it increases the risk of error. We therefore developed this mechanism to be small enough to avoid fatal errors in operation. The diameter of the first prototype is $6 \mathrm{~mm}$, and the minimum radius of curvature of bending is about $8.5 \mathrm{~mm}$. 


\subsection{Mechatoronical Control}

This bending forceps (Forceps with Bending Mechanism) needs very large forces driving the wires to generate similar forces on the tip of the forceps (bending and grasping forces). Moreover single-handed manual control of three DOF (two bending and one grasping) is very difficult and inadequate for this forceps. This is why we use mechatronic components for this bending forceps to drive the bending and grasping DOF.

The motors, which cannot be sterilized, are placed sufficiently far from the surgical field for maintenance of sterility, and stainless steel wires transmit the forces produced by the motors. In this system, since there are no electronic components (motors, sensors, etc.) on the tip of the forceps manipulator (which is inserted into the patient), this manipulator is perfectly sterilizable. This arrangement, with remote electronic components, and the Multi-DOF forceps manipulator system, which is lightweight, simple, and perfectly clean, can easily be assembled (Fig. 6).

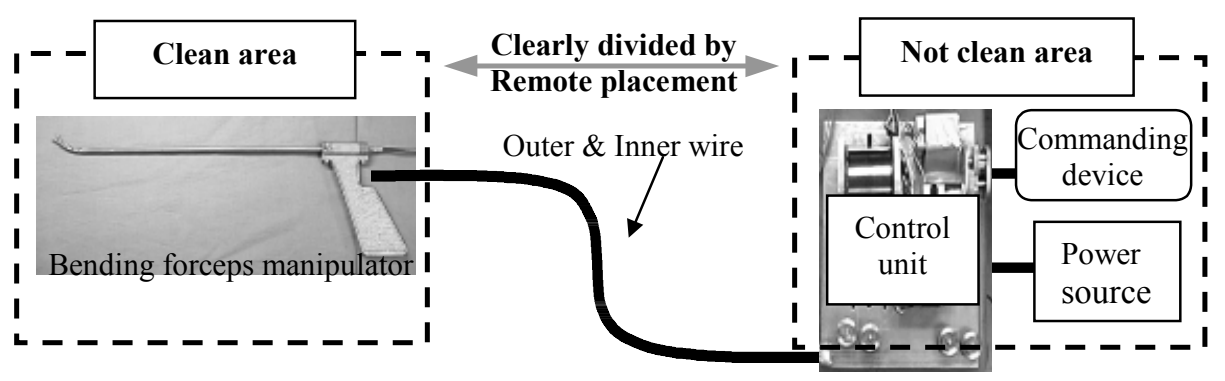

Fig. 6. Total system components of the Multi-DOF forceps manipulator.

Fig. 7. A photograph of the first prototype manipulator. The characteristics of the manipulator are presented in Table 1.

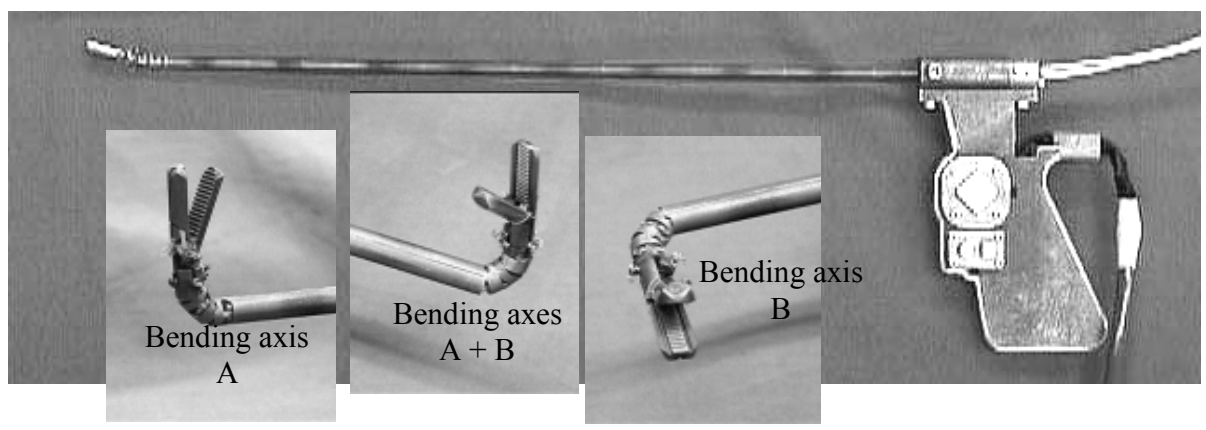

Fig. 7. First Prototype of Multi-DOF forceps manipulator 
Table 1. Specification of the bending forceps manipulator system

\begin{tabular}{cc}
\hline $\begin{array}{c}\text { Range of actions (DOF) } \\
\text { Moving range }\end{array}$ & 2 bending and 1 grasping \\
Diameter & $0-90^{\circ}$ (bending) $/ 0-60^{\circ}$ (grasping) \\
Size & $6 \mathrm{~mm}$ \\
Weight & $455 \mathrm{~mm}(1) \times 130 \mathrm{~mm}(\mathrm{~h}) \times 15 \mathrm{~mm}(\mathrm{~d})$ \\
Material & $270 \mathrm{~g}($ Multi-DOF forceps) \\
\end{tabular}

\section{Experimental Results}

\subsection{Mechanical Characteristics}

Firstly, we checked the characteristics of bending movements. The bending angles are shown in Fig. 8 and 9. The movement characteristics have hysteresis, with large backlash due to the stretching and friction of the wires. The difference between bending drive and extension drive (on drive speed and backlash) is mainly caused by the difference in the driving forces of wire (for bending and for extension). The difference between bending axes A and B may be caused by the differences in the loss of drive power, due to the different placement of the hinges of each axis. The accuracy of the movement is within 4.3 degrees for each axis.

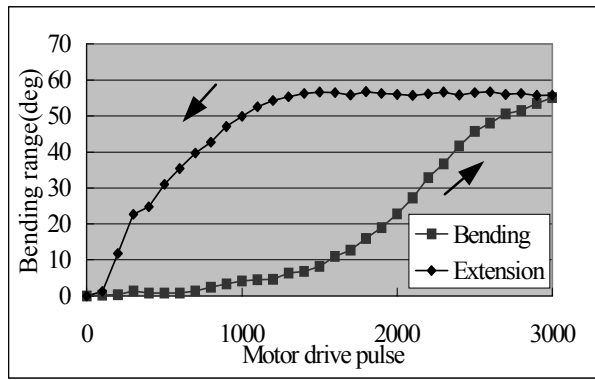

Fig. 8. Movement of Bending axis A

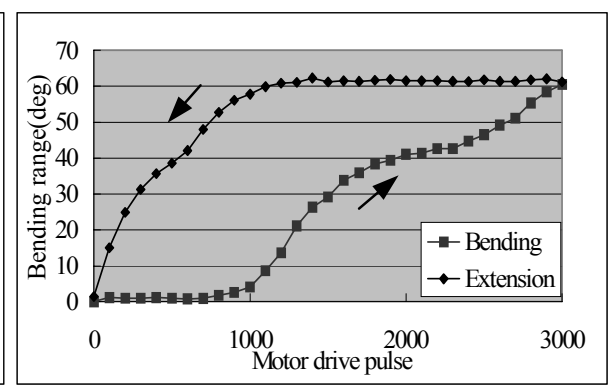

Fig. 9. Movement of Bending axis B

The generated forces, bending and grasping, are measured using weights. The results are shown in Table 2. The generated forces of bending (driving and holding) and grasping are sufficient, but the forces of extension are notably inadequate. This is because of the low power of the spring that controls the tension of the wire for extension.

Table 2. Generated force of Multi-DOF forceps manipulator

\begin{tabular}{|c|c|c|}
\hline & \multicolumn{2}{|c|}{ Generated force } \\
\hline & Driving & Holding \\
\hline Bending & $0.45 \mathrm{kgf}$ & $0.50 \mathrm{kgf}$ \\
\hline Extension & $0.05 \mathrm{kgf}$ & $0.08 \mathrm{kgf}$ \\
\hline Grasping & \multicolumn{2}{|c|}{$0.85 \mathrm{kgf}$} \\
\hline
\end{tabular}




\subsection{In vivo Testing}

We tested the clinical performance of this Multi-DOF forceps manipulator by using it in a semi-clinical situation. The surgeon carried out trial operations (grasping, bending, extension, etc.) around the liver after ordinary laparoscopic cholecystectomy on a pig (Fig. 10 and 11).
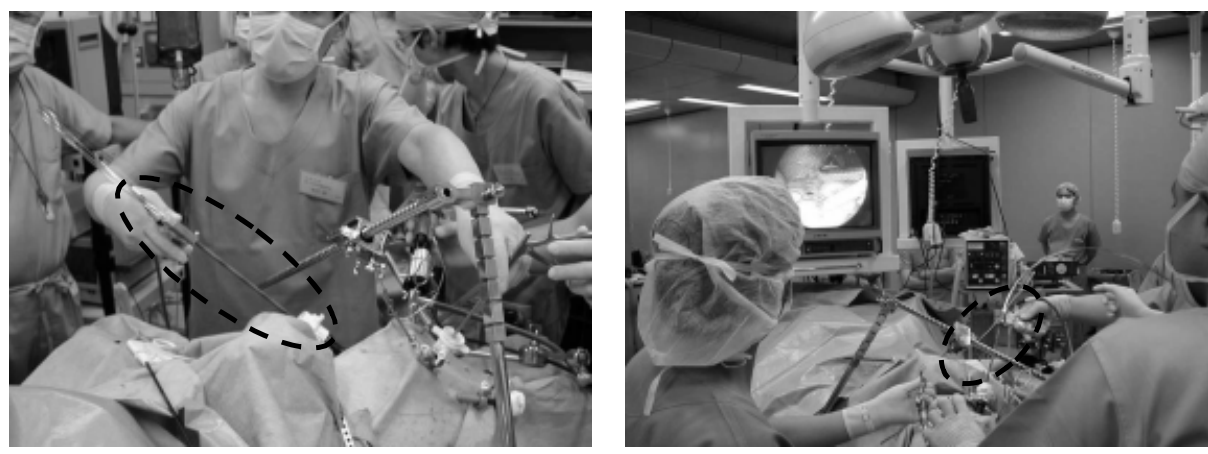

Fig. 10. In vivo testing of the Multi-DOF forceps manipulator system (overview)

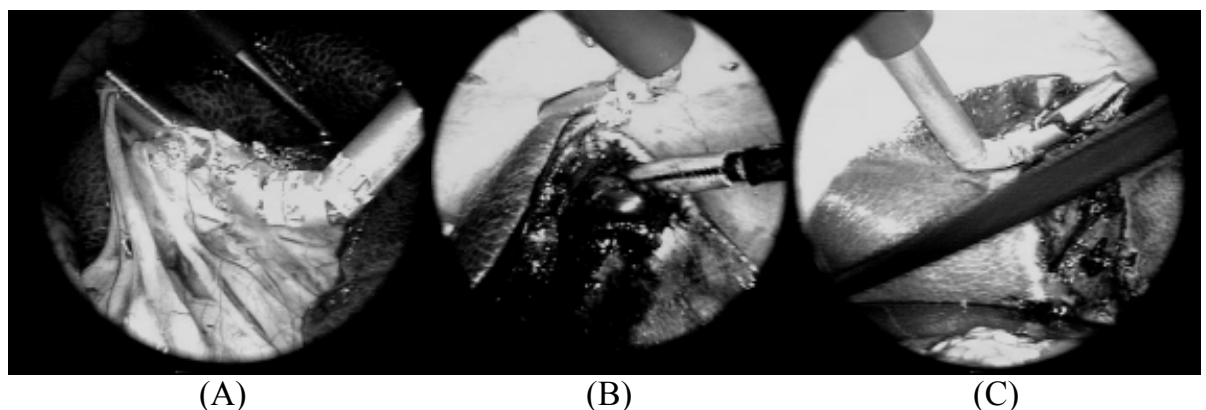

(A)

(B)

(C)

Fig. 11. In vivo testing of the Multi-DOF forceps manipulator system (laparoscopic view)

The results show that the bending force (driving and holding), the grasping force, and the radius of curvature of bending are nearly adequate. "User-friendliness", which mainly depends on the size, weight, and man-machine interface of this forceps manipulator, is satisfactory.

Using the Multi-DOF forceps manipulator, an operation that is impossible using current straight, rigid forceps, was carried out. In Fig. 11(B), although inserted into the visceral cavity through the left upper trochar, the manipulator works as if it had been inserted through the right upper trochar. In Fig. 11(C), the bent part of the forceps was effectively used to control the position of the liver (pushing). However, the extension force (driving and holding) was insufficient, and the stick-slip movement appeared because of this. The poor responses of movement (due to the backlash of the wire drive system) are not a problem in bending and extension. However, in grasping, a high response of movement is needed. We plan to solve these 
problems in extension forces and the response of grasping movements in the next prototype.

In this experiment, although there were indeed problems caused by the extension drive, the basic functions of this Multi-DOF forceps manipulator were confirmed as sufficiently useful for laparoscopic surgery.

\section{Discussion}

\subsection{Multi-DOF Forceps Manipulator}

Using simple and rigid components to achieve a bending mechanism, this manipulator established a high level of performance, with large output based on small components. The remote placement of electronic components ensured the clean, simple and lightweight nature of the Multi-DOF forceps manipulator. In the near future, this equipment promises to overcome the problems with current forceps and will raise the standards of laparoscopic surgery with higher effectiveness and safety.

The most important problems of this manipulator are that of extension force and response of movement. However, this problem may be solved if we can develop intelligent control mechanisms for force sensing and feedback systems.

\subsection{Future Work New System Concept}

The conventional laparoscope, with a rigid scope, provides a limited and small workspace to the surgeon. Flexible laparoscopes solve this problem in part, but controlling the line of sight in a flexible laparoscope is more complex. The new forceps manipulator we are developing now integrates the bending forceps with a fiberscope (Fig. 12). Using this manipulator, the surgeon can see the workspace from two different lines of sight (one is that of the laparoscope, and the other is that of the fiberscope) without controlling the view-line of an additional endoscope (fiberscope). We think surgeons can efficiently operate using both a laparoscope view ("macro" view) and a fiberscope view ("micro" view). Especially when operating on the far side of organs, the fiberscope view will be very helpful for surgeons.

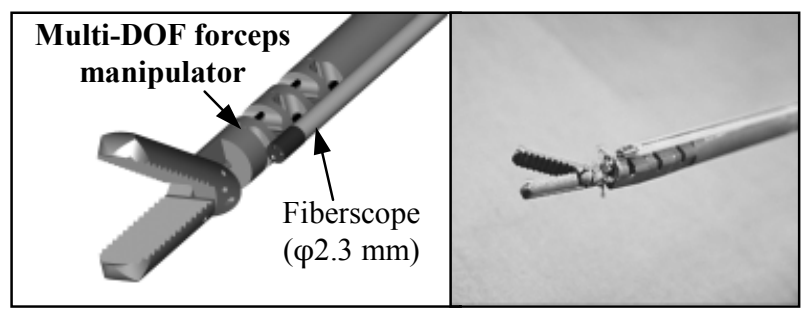

Fig. 12. New Multi-DOF forceps manipulator integrated with a fiberscope

Using the Multi-DOF forceps manipulator, we will construct a new surgical manipulator system concept. We have already developed a laparoscope manipulator using a five-bar linkage system [7]. Integrating the forceps manipulator and five- 
linkage manipulator, we will develop new surgical manipulators for laparoscopic surgery. Both of these system components (Multi-DOF forceps manipulator and fivebar linkage manipulator) were developed to achieve safety and reliability.

\section{Conclusion}

We have developed a Multi-DOF forceps manipulator with bending mechanism for laparoscopic surgery. This mechanism achieved two additional DOF of bending, with small radius and a large driving force on the tip of the slim forceps. Through the in vivo testing, although some problems were found, the effectiveness of this manipulator was clearly demonstrated. This manipulator can solve many problems in laparoscopic surgery due to the poor DOF of controlling surgical tools. We are currently developing a new surgical manipulator for laparoscopic surgery using this Multi-DOF forceps manipulator.

\section{Acknowledgements}

This study was partly supported by the Research for the Future Program JSPS-RFTF 96P00801 and JSPS-RFTF 99I00904.

\section{References}

1. Rininsland H.: ARTEMIS. A telemanipulator for cardiac surgery, European Journal of Cardio-Thoracic Surgery 16(2) (1999) 106-111

2. Shennib H., Bastawisy A., McLoughlin J., Moll F.: Robotic computer-assisted telemanipulation enhances coronary artery bypass, Journal of Thoracic \& Cardiovascular Surgery 117(2) Feb (1999) 310-313

3. Cohn, M., L. S. Crawford, J. M. Wendlandt, S. S. Sastry: Surgical Applications of MilliRobots, Journal of Robotic Systems 12(6) (1995) 401-416

4. Steve Charles, Hari Das, Tim Ohm, Curtis Boswell, Guillermo Rodriguez, Robert Steele, Dan Istrate: Dexterity-enhanced Telerobotic Microsurgery Proceedings of 8th International Conference on Advanced Robotics (ICAR `97) (1997) 5-10

5. Ikuta K., Kato T., Nagata S.: Micro active forceps with optical fiber scope for intra-ocular microsurgery, Proceedings of the IEEE Micro Electro Mechanical Systems (MEMS) (1996) 456-461

6. Gupta V., Reddy NP., Batur P.: Forces in Laparoscopic Surgical Tools, Presence 6(2) April (1997) 218-228

7. Kobayashi E. et al.: Development of a laparoscope manipulator using five-bar linkage mechanism, CAR97 Computer Assisted Radiology and Surgery - Proceeding of the $11^{\text {th }}$ International Congress and Exhibition - (1997) 825-830

8. R. Nakamura et al.: Development of forceps manipulator system for laparoscopic surgery, CARS2000 Computer Assisted Radiology and Surgery - Proceeding of the $14^{\text {th }}$ International Congress and Exhibition - (2000) 105-110

9. Hashimoto D.: Gasless Laparoscopic Surgery World Scientific Publishing (1995) 